\title{
ATF4 regulates lipid metabolism and thermogenesis
}

\author{
Chunxia Wang ${ }^{1}$, Zhiying Huang ${ }^{1}$, Ying $\mathrm{Du}^{1}$, Ying Cheng ${ }^{1}$, Shanghai Chen ${ }^{1}$, Feifan Guo ${ }^{1}$ \\ ${ }^{1}$ Key Laboratory of Nutrition and Metabolism, Institute for Nutritional Sciences, Shanghai Institute for Biological Sciences, Chi- \\ nese Academy of Sciences, The Graduate School of the Chinese Academy of Sciences, 294 Taiyuan Road, Shanghai 200031, China
}

Activating transcription factor 4 (ATF4) has been shown to play key roles in many physiological processes. There are no reports, however, demonstrating a direct link between ATF4 and lipid metabolism. We noticed that Atf4deficient mice are lean, suggesting a possible role for ATF4 in regulating lipid metabolism. The goal of our current study is to investigate the involvement of ATF4 in lipid metabolism and elucidate the underlying mechanisms. Studies using Atf4-deficient mice revealed increased energy expenditure, as measured by oxygen consumption. These mice also showed increases in lipolysis, expression of uncoupling protein 2 (UCP2) and $\beta$-oxidation genes and decreases in expression of lipogenic genes in white adipose tissue (WAT), suggesting increased utilization and decreased synthesis of fatty acids, respectively. Expression of UCP1, 2 and 3 was also increased in brown adipose tissue (BAT), suggesting increased thermogenesis. The effect of ATF4 deletion on expression of UCPs in BAT suggests that increased thermogenesis may underlie increased energy expenditure. Thus, our study identifies a possible new function for ATF4 in regulating lipid metabolism and thermogenesis.

Keywords: ATF4, lipid metabolism, thermogenesis, WAT, BAT

Cell Research (2010) 20:174-184. doi: 10.1038/cr.2010.4; published online 12 January 2010

\section{Introduction}

White adipose tissue (WAT) plays an active role in energy balance, because it not only serves as a lipid storing tissue but also as an endocrine organ secreting adiposederived hormones [1]. Lipid metabolism in WAT, including lipogenesis, lipolysis and fatty acid oxidation, is under tight control of the sympathetic nervous system (SNS) and adipose-derived hormones [2]. The mass of WAT is determined by a balance between energy intake and energy expenditure [3]. Total energy expenditure includes physical activity, basal metabolism and adaptive thermogenesis. It has been well established that thermogenesis represents a major form of energy expenditure and plays a significant role in the maintenance of energy balance [3]. Brown adipose tissue (BAT) is the primary site for thermogenesis and functions to regulate body weight by increasing the expression of uncoupling protein 1 (UCP1)

Correspondence: Feifan Guo

Tel: +86-21-54920250; Fax: +86-21-54920291

E-mail: ffguo@sibs.ac.cn

Received 15 October 2009; revised 4 November 2009; accepted 20 November 2009; published online 12 January 2010 in rodents $[4,5]$. Activation of UCP1 increases uncoupling of oxidative respiration from ATP synthesis, resulting in dissipation of energy as heat [5-7]. This function of UCP1 is responsible for normal energy balance, because UCP1 ablation induces obesity [8]. Transcriptional regulation of the $U_{c p} 1$ gene is positively controlled by several transcription factors including members of the peroxisome proliferators-activated receptor (PPAR) and CCAAT/enhancer-binding protein (C/EBP) families and cAMP response element-binding protein (CREB)[9]. Recently, it has been shown that UCP1 expression can also be negatively regulated by the liver X receptor [10].

Activating transcription factor 4 (ATF4), also known as CREB2, TAXREB67, mTR67 or C/ATF, belongs to the family of basic zipper-containing proteins [11-14]. It has been shown that ATF4 is constitutively expressed in a wide variety of tissues including brain, heart, WAT, BAT, liver, spleen, thymus, lung and kidney, as well as in cell lines derived from $T$ cells, B cells, monocytes and fibroblasts $[14,15]$. Accumulated evidence suggests that ATF4 plays an important role in regulation of the highlevel proliferation required during fetal-liver hematopoiesis [16], long-term memory [17-19], osteoblast differentiation [20], ER stress [21, 22], amino acid deprivation [23] and glucose metabolism [24], etc. It has also been 
reported that ATF4 plays a key role in thermogenesis in BAT in small mammals [15]. There has been no report, however, that demonstrates a direct link between ATF4 and lipid metabolism.

It has previously been reported that $\mathrm{Atf4}^{-/-}$mice are small [16]. We noticed that these mice were lean, suggesting that ATF4 plays a role in lipid metabolism. The physiological role and significance of ATF4 in lipid metabolism and thermoregulation were therefore investigated in our work. We observed increase in lipolysis and expression of $\beta$-oxidation genes, and decrease in expression of lipogenic genes in WAT, consistent with increased utilization and decreased synthesis of fatty acids, respectively. In addition, we observed increases in expression of UCP1 in BAT, suggesting increased thermogenesis, consistent with increased energy expenditure as measured by oxygen consumption. We hypothesize that these changes in WAT and BAT account for the significantly reduced fat mass in $\mathrm{Atf}^{-/-}$mice.

\section{Results}

Reduced WAT mass in Atf ${ }^{-/}$mice

Apart from being smaller compared with wild-type mice during postnatal development (Figure 1A), the surviving $\mathrm{AtfH}^{-/-}$mice appeared healthy. Weight of body and tissues, including WAT and BAT, was markedly reduced in male $\mathrm{Atf4}^{-/-}$mice (Figures 1A and 1B). Consistent with these changes, the adiposity index (ratio of abdominal body fat to body weight) was also reduced $(0.90 \%$ vs $0.17 \%, P<0.01$ ) (Figure 1B). By contrast, the weight of tissues, including heart, liver and kidney, was similar in male $\mathrm{Atf4}^{-/-}$and $\mathrm{Atf4^{+/+ }}$ mice (Figure 1B). The growth curve and body weight in both males and females, at all ages examined, were similar between $\mathrm{AtfH}^{+/-}$and $\mathrm{Atf4^{+/+ }}$ mice (data not shown). We also found that $A t f 4^{-/-}$mice are resistant to the high-fat diet-induced obesity, as shown by the decreased body and WAT weight compared with those of wild-type mice (data not shown).

Serum parameters were also analyzed in $\mathrm{Atf}^{+/+}$and Atf $4^{-/-}$mice (Table 1). There were no differences in levels of serum cholesterol between the male $A t f 4^{+/+}$and $A t f 4^{-/-}$mice. By contrast, levels of serum triglyceride (TG) and free fatty acids were much lower in Atf4 ${ }^{-1}$ mice compared to $\mathrm{AtfH}^{+/+}$mice. Consistent with the decreased WAT mass in $\mathrm{Atf}^{-1-}$ mice, levels of leptin decreased by about $83 \%$ in these mice. Levels of serum Norepinephrine (NE) were increased in $\mathrm{Atf}^{-/-}$mice. There were, however, no differences in serum thyroid hormone 3,5,3'-triiodothyronine (T3) and Epinephrine

Table 1 Serum measurements in $\mathrm{AtfH}^{+/+}$and $\mathrm{AtfH}^{-/-}$mice

\begin{tabular}{|c|c|c|}
\hline Parameter & Atf $4^{+/+}$mice & Atf $4^{-1-}$ mice \\
\hline Serum cholesterol (mg/dl) & $140.8 \pm 8.7$ & $120.1 \pm 12.1$ \\
\hline Serum triglyceride $(\mathrm{mg} / \mathrm{dl})$ & $210.9 \pm 40.8$ & $119.5 \pm 21.1^{*}$ \\
\hline Serum free fatty acid $(\mu \mathrm{M})$ & $611.3 \pm 65.1$ & $436.5 \pm 23.2 *$ \\
\hline Insulin (ng/ml) & $0.36 \pm 0.03$ & $0.33 \pm 0.01$ \\
\hline Glucose (mg/dl) & $146.3 \pm 5.0$ & $146.1 \pm 3.1$ \\
\hline Leptin $(\mathrm{pg} / \mathrm{ml})$ & $816.1 \pm 194.7$ & $141.0 \pm 40.6^{*}$ \\
\hline $\mathrm{T} 3(\mathrm{pmol} / \mathrm{l})$ & $23.3 \pm 5.9$ & $24.5 \pm 3.2$ \\
\hline Norepinephrine (ng/l) & $227.6 \pm 22.0$ & $267.1 \pm 29.6^{* *}$ \\
\hline Epinephrine $(\mu \mathrm{g} / \mathrm{l})$ & $122.6 \pm 6.8$ & $134.6 \pm 8.8$ \\
\hline
\end{tabular}

Numbers of mice (2-3-months-old) used: $n=8$ in each group. Data are mean \pm SEM of at least two independent experiments. Statistical significance was determined by two-tailed student $t$-test $(* P<0.05$; **P $<0.01$ ).
A

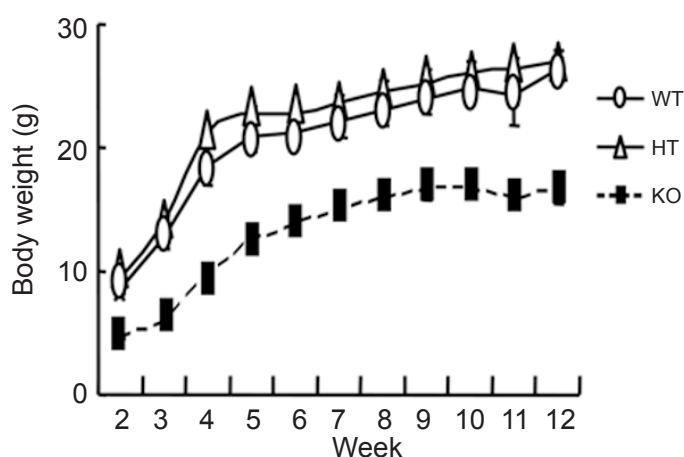

B

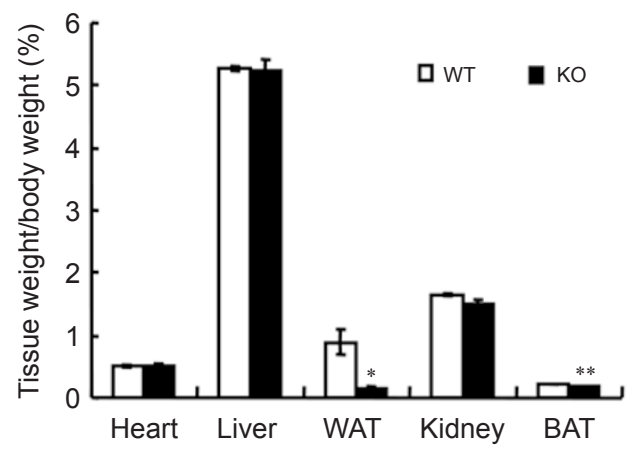

Figure 1 Body weight and fat mass decreases in Atf ${ }^{-/-}$mice. Data are mean \pm SEM of at least two independent experiments $\left(A t f 4^{+/+}\right.$mice $(n=8), A_{t f 4^{-/}}$mice $\left.(n=8)\right)$. Statistical significance was determined by two-tailed student $t$-test $(* P<0.05$, $* * P<$ 0.01). (A) Growth curve. (B) Tissue mass in proportion to body weight. 
(EPI) levels between $\mathrm{AtfH}^{+/+}$and $\mathrm{Atf4}^{-/-}$mice. Furthermore, there were no differences in serum levels of insulin and glucose. Similar changes of these serum parameters were also observed in female ttf $^{-/-}$mice compared with their wild-type controls (data not shown). Based on the similar phenotypes between male and female $\mathrm{Atf4}^{-/-}$ mice, male $\mathrm{Atf4}^{-/-}$mice were chosen for the following experiments in this study.

\section{Increased oxygen consumption in Atf4 ${ }^{-/-}$mice}

The reduced WAT in $\mathrm{Atf4}^{-/-}$mice could result from an imbalance between food intake and energy expenditure. Daily food intake, however, was not decreased in $\mathrm{Atf4}^{-}$ compared with $A t f 4^{+/+}$mice. By contrast, when normalized by body weight, $\mathrm{Atf4}^{-/-}$mice consumed even $35 \%$ more food than $\mathrm{AtfH}^{+/+}$mice (Figure 2A). To determine whether the reduced fat mass of $A t f 4^{-/}$mice could be a consequence of an increase in energy expenditure, we measured oxygen consumption by indirect calorimetry. The total energy expenditure ( $24 \mathrm{~h} \mathrm{O}_{2}$ consumption) was markedly increased in $\mathrm{Atf4}^{-/-}$compared with $\mathrm{Atf4}^{+/+}$mice
(Figures $2 \mathrm{~B}$ and $2 \mathrm{C}$ ). The respiratory exchange ratio (RER, $\mathrm{VCO}_{2} / \mathrm{VO}_{2}$ ) was lower in these mice (Figures $2 \mathrm{D}$ and $2 \mathrm{E}$ ).

Because of the continuously high oxygen consumption in $\mathrm{Atf4}^{-/-}$mice, we assessed energy expenditure from different aspects to determine the possible cause for it. We did not see a difference in locomotor activity between Atf $4^{-/}$and $A t f 4^{+/+}$mice (data not shown). We therefore speculated that increased thermogenesis might account for increased energy expenditure in $\mathrm{Atf4^{-/ }}$ mice. As expected, we found that $A t f 4^{-1-}$ mice maintained higher core temperatures throughout the 24-h period compared with Atf $4^{+/+}$mice (Figure 2F).

\section{Reduced WAT cell volume in Atf4 ${ }^{-/-}$mice}

The reduced adipose mass in $A t f 4^{-/-}$mice may result from decreased adipocyte volume and/or number. Histological analysis of WAT showed that loss of ATF4 expression resulted in a $45 \%$ reduction in adipocyte volume compared with $A t f 4^{+/+}$mice (Figure 3A). However, cell numbers were the same in both $\mathrm{AtfH}^{+/+}$and $\mathrm{Atf4^{-/ }}$ mice, as demonstrated by DNA content analysis (data not shown).

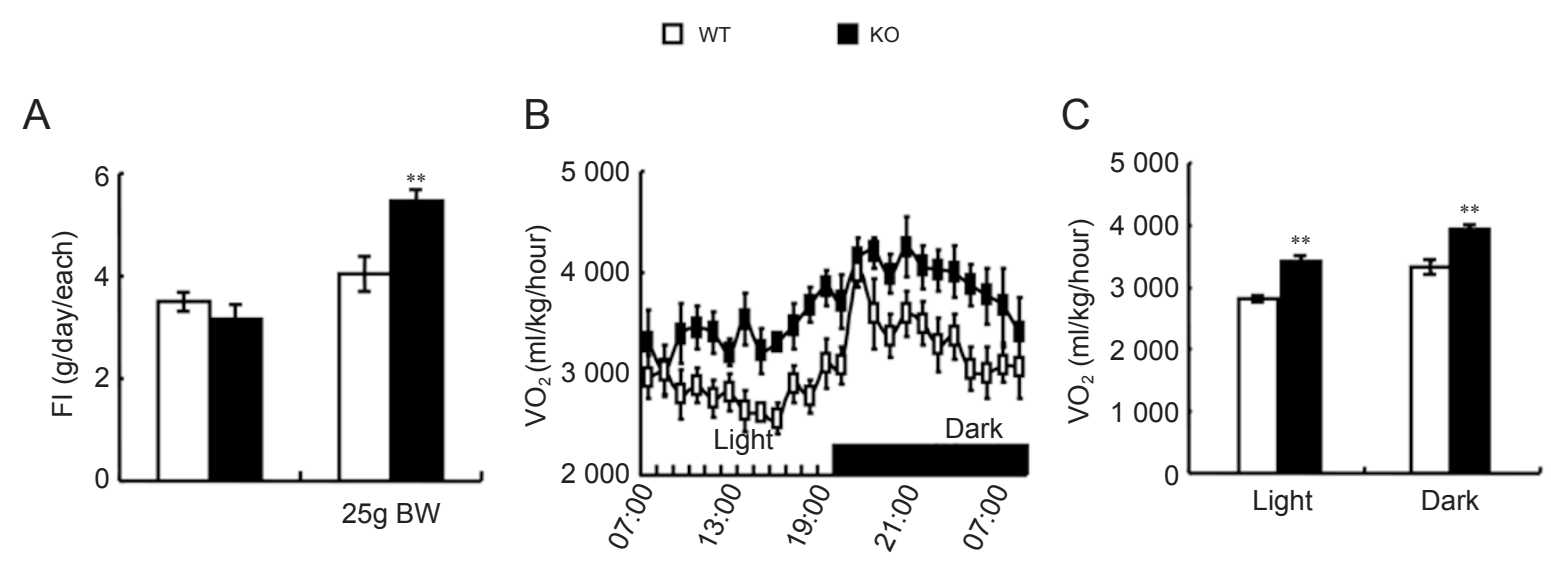

$\mathrm{D}$

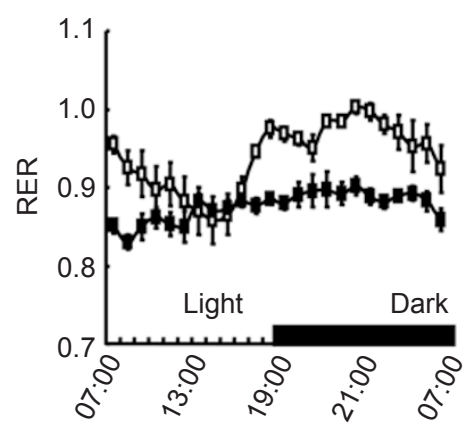

E

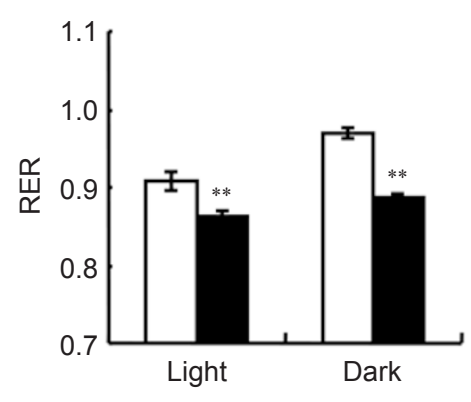

$\mathrm{F}$

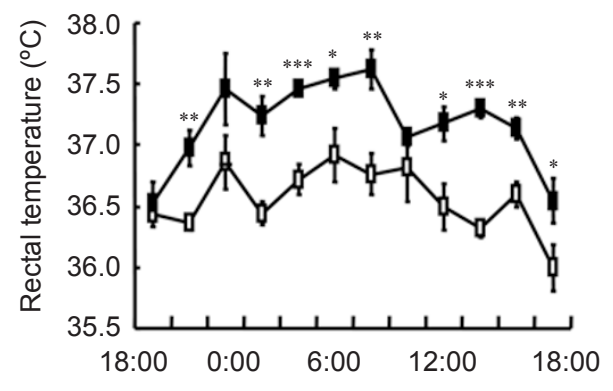

Figure 2 Energy expenditure increases in Atf4 ${ }^{-/-}$mice. (A) Food intake. (B and C) 24-h oxygen consumption. (D and E) Respiratory exchange ratio (RER); (F), Rectal temperature. Data are mean \pm SEM of at least two independent experiments $\left(A t f 4^{+/+}\right.$mice $(n=8)$, Atf $4^{-1-}$ mice $\left.(n=8)\right)$ over $24-48 \mathrm{~h}$ after $6 \mathrm{~h}$ acclimation to the metabolic chamber. Statistical significance was determined by two-tailed student's $t$-test $(* P<0.05, * * P<0.01, * * * P<0.001)$. 
Repressed lipogenesis in WAT of Atf $4^{-/-}$mice

The reduced fat mass in $A t f 4^{-/-}$mice likely reflects an imbalance in TG synthesis, lipolysis and fatty acid $\beta$-oxidation. For this reason, we examined changes in expression levels of mRNAs coding for proteins related to each of these processes. We first investigated genes underlying the synthesis of TGs, which include fatty acid synthase (FAS), stearoyl CoA desaturase 1 (SCD1) and sterol regulatory element-binding protein 1c (SREBP1c). Levels of $F$ as and $S c d 1$ mRNA were significantly reduced in WAT of $\mathrm{Atf}^{-/-}$compared with Atf4 ${ }^{+/+}$mice (Figure 3B). Consistent with decreased Fas mRNA expression, levels of FAS protein were also decreased by $40 \%$ in $\mathrm{Atf} 4^{-/}$ mice (Figure 3C). Furthermore, levels of SREBP1c, the major transcription factor regulating lipogenesis in WAT, were also markedly decreased in $\mathrm{Atf4^{-/- }}$ mice (Figure 3C). Very interestingly, expression of Srebplc mRNA was not decreased in WAT of $\mathrm{Atf}^{-/-}$mice (Figure 3B).
Enhanced lipolysis in WAT of Atf4 $4^{-/-}$mice

To investigate whether the diminished adipocyte volume observed in $\mathrm{Atf4}^{-/-}$mice could also result from increased TG lipolysis and/or fatty acid $\beta$-oxidation, we examined changes in levels of phosphorylated proteins related to each of these processes. We first investigated whether levels of hormone-sensitive lipase (HSL), the rate limiting enzyme for TG lipolysis, were differentially regulated in WAT of $\mathrm{Atf4}^{-/-}$and $\mathrm{Atf4^{+/+ }}$ mice. Levels of total HSL were not significantly different in WAT between $\mathrm{Atf4}^{-/-}$and $\mathrm{Atf4}^{+/+}$mice. By contrast, levels of phosphorylated HSL (p-HSL) were significantly increased in WAT of $\mathrm{Atf4}^{-1-}$ mice compared with $\mathrm{Atf}^{+/+}$ mice (Figure 4A). Consistent with increased levels of p-HSL, levels of phosphorylated substrate for PKA, the kinase that phosphorylates HSL, were also elevated in WAT of $\mathrm{AtfH}^{-/}$mice (Figure 4A). Expression levels of Atgl and Perilipin A, which encode proteins regulating

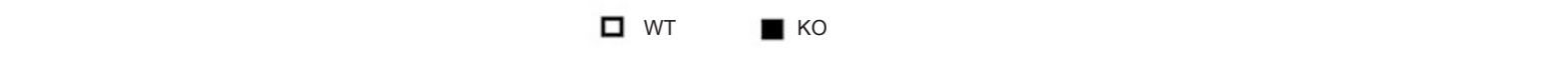

A
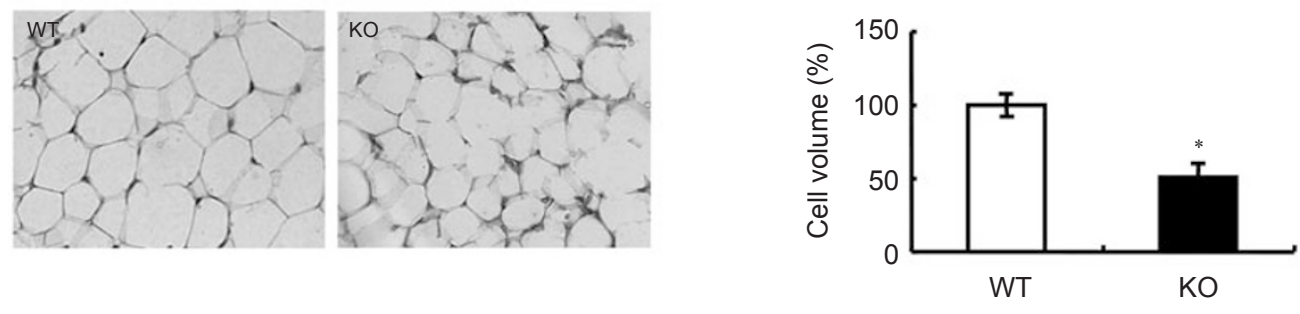

B

C
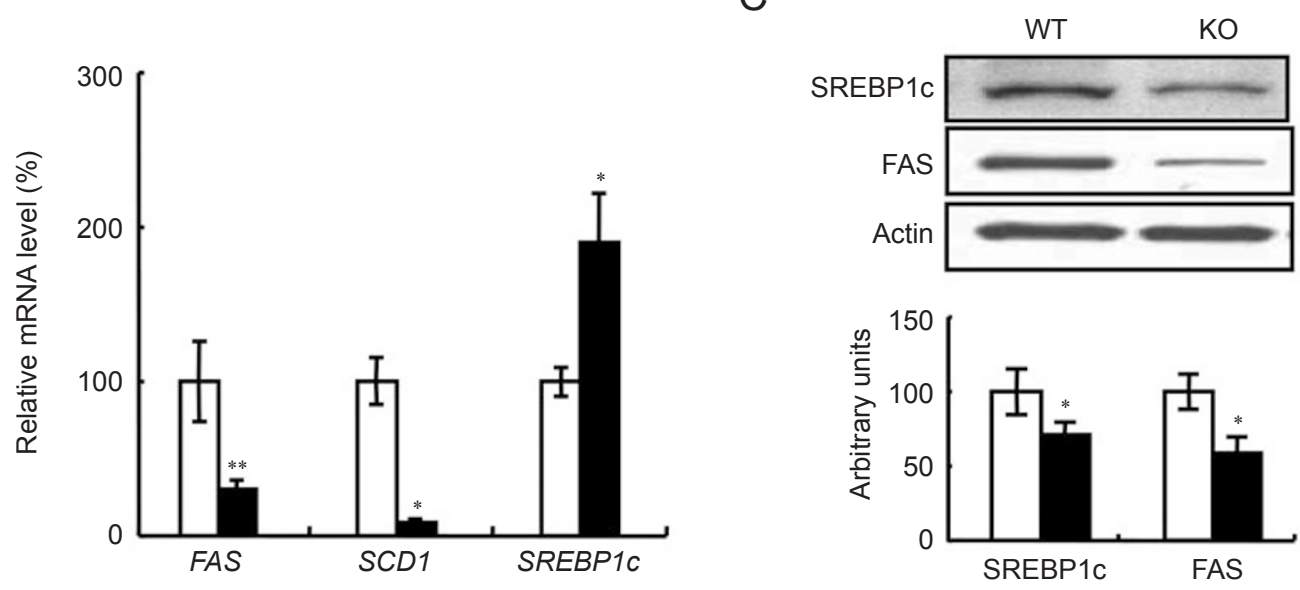

Figure 3 Lipogenic genes represses in WAT of $A t f 4^{-1-}$ mice. (A) WAT sections from mice in each group were stained with hematoxylin and eosin ( $\times 20$ magnification). Representative images of several animals for each group (left) and analysis of WAT cell volume (right). Expression of lipogenic genes and proteins. (B) FAS, SCD1 and SREBP1C mRNAs. (C) SREBP1C and FAS proteins (top, western blot; bottom, protein levels relative to Actin). Data are mean \pm SEM of at least two independent real-time PCR experiments or western blot with mice, as indicated $\left(\right.$ Atf $4^{+/+}$mice $(n=8)$, Atf $^{-/-}$mice $\left.(n=8)\right)$. Statistical significance was determined by two-tailed student $t$-test $(* P<0.05, * * P<0.01)$. 


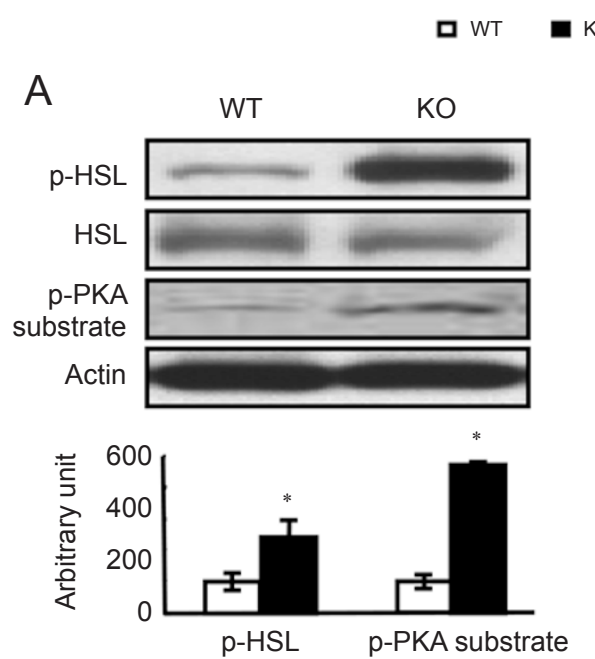

C

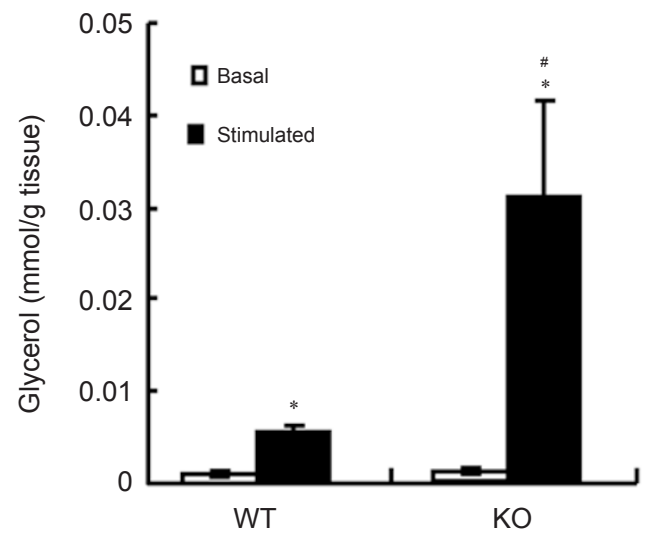

E

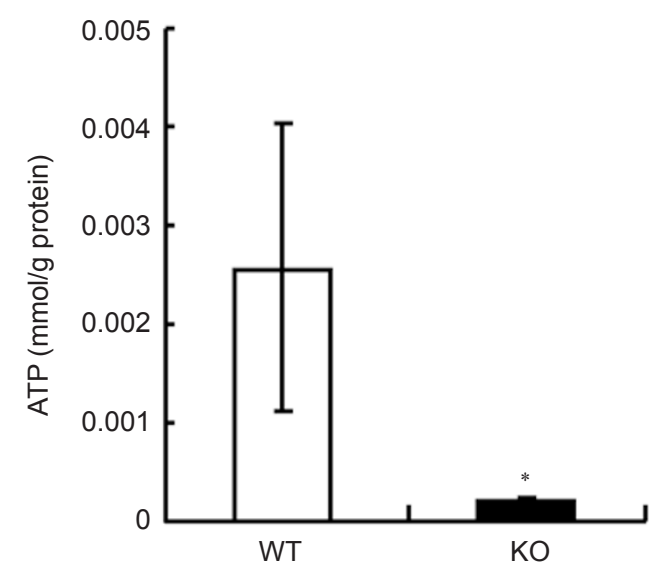

B

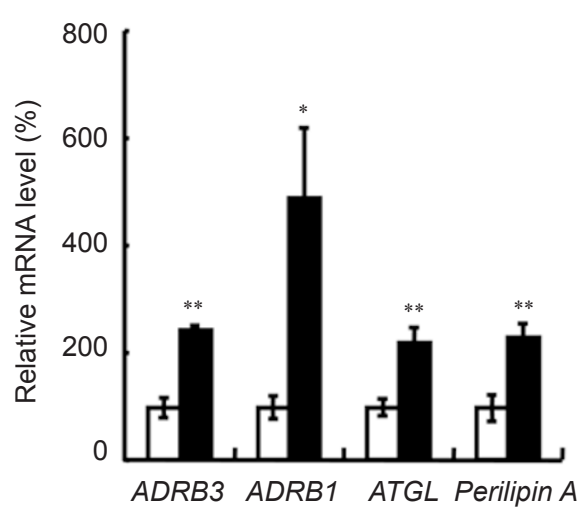

D

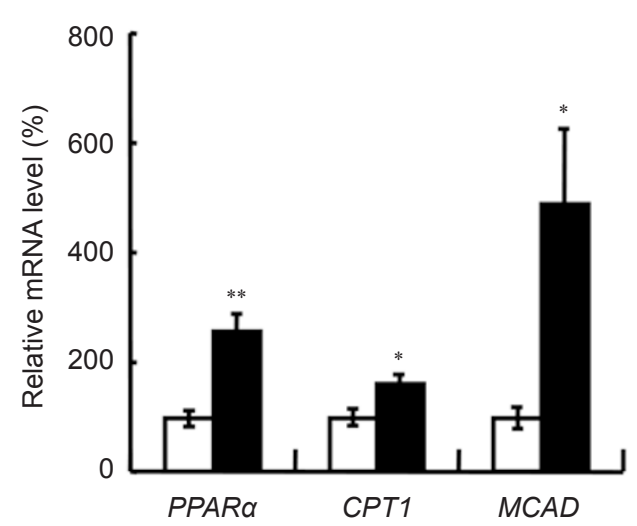

$\mathrm{F}$

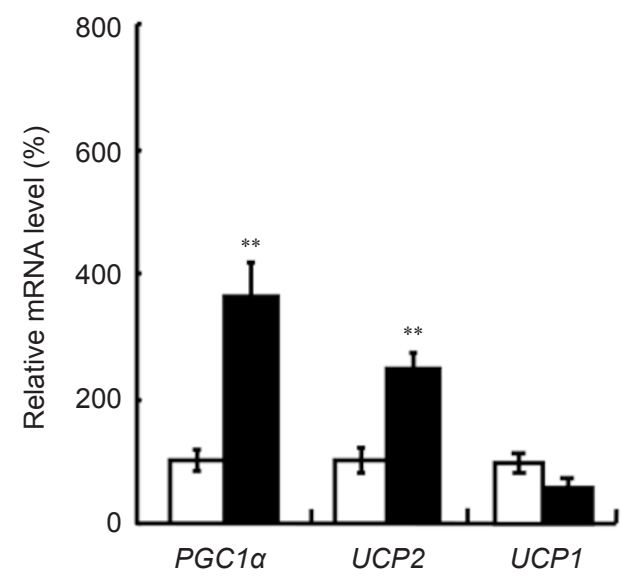

Figure 4 TG lipolysis and fatty acid $\beta$-oxidation genes increase in WAT of Atf4 ${ }^{-/-}$mice. Data are mean \pm SEM of at least two

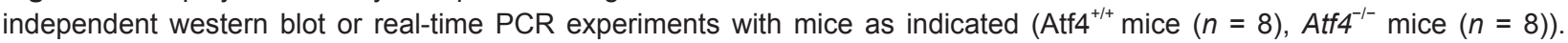
Statistical significance was determined by two-tailed student $t$-test $\left({ }^{*} P<0.05\right.$, $\left.* * P<0.01\right)$. (A) $\mathrm{p}$-HSL and HSL proteins (top, western blot; bottom, p-HSL relative to HSL). (B) Adrb3, Adrb1, ATGL and Perilipin A mRNA. (C) Glycerol release assay $\left({ }^{*} P<0.05\right.$, basal level vs stimulated level; \#P < 0.05 WT vs KO); (D) PPAR,$C P T 1$ and MCAD mRNA; (E) ATP levels; (F) $P G C 1 \alpha, U C P 2$ and UCP1 mRNA. 
lipolysis, were significantly increased in WAT of $A t f 4^{-/}$ compared with $\mathrm{AtfH}^{+/+}$mice (Figure 4B). To further investigate the effect of ATF4 on lipolysis, we compared basal and stimulated glycerol release in WAT isolated from $\mathrm{AtfH}^{+/+}$and $\mathrm{Atf4^{-/ }}$ mice. The rates of glycerol release following stimulation with the $\beta$-adrenoceptor agonist isoproterenol were significantly higher in WAT isolated from $\mathrm{Atf4}^{-/-}$compared with $\mathrm{Atf4} 4^{+/+}$mice; however, there were no significant differences in glycerol release at basal level (Figure 4C). Increased lipolysis in these mice was accompanied with increased expression of $\beta$-adrenoceptor 3 (Adrb3) and Adrb1 mRNAs (Figure 4B).

Increased expression of $\beta$-oxidation-related genes in WAT of Atf $4^{-1-}$ mice

To examine if genes and proteins related to fatty acid $\beta$-oxidation are differentially regulated in WAT of $A t f 4^{+/+}$ and $\mathrm{Atf4}^{-/-}$mice, we examined the expression levels of mRNAs for the transcription factor PPAR $\alpha$ and its target genes carnitine palmitoyltransferase 1 (CPT1) and medium-chain acyl-CoA dehydrogenase (MCAD). Expression of these genes was significantly increased in WAT of $\mathrm{Atf4}^{-/-}$compared with $\mathrm{Atf4^{+/+ }}$ mice (Figure 4D). Despite increased expression of $\beta$-oxidation-related genes, levels of ATP in WAT were very much lower in $\mathrm{Atf}^{-/}$compared with $\mathrm{Atf4}^{+/+}$mice (Figure 4E), which is associated with significantly increased expression of peroxisome proliferators-activated receptor gamma coactivator 1 (Pgcla) and Ucp2 (Figure 4F). We did not see difference in UCP1 expression in WAT between $\mathrm{Atf4}^{-/-}$and $\mathrm{Atf4^{+/+ }}$ mice (Figure 4F). Furthermore, the expression levels of UCP3 were lower in WAT of $\mathrm{Atf}^{-/-}$compared with $\mathrm{Atf4}^{+/+}$mice (data not shown).

Enhanced expression of fatty acid oxidation genes in BAT of Atf4 ${ }^{-/-}$mice

Many studies have demonstrated that increased lipolysis increases levels of FFA in serum. Despite increased lipolysis, levels of FFA in serum were low in $\mathrm{Atf4}^{-/-}$mice (Table 1), suggesting possibly increased fatty acid utilization by other tissues, including liver, BAT and muscle. Expression of genes related to $\beta$-oxidation, including Ppara, Cpt1 and Mcad, however, was not upregulated in livers and muscles of $\mathrm{AtfH}^{-/-}$compared with $\mathrm{Atf}^{+/+}$mice (Supplementary information, Figures S1 and S2). By contrast, fatty acid $\beta$-oxidation-related genes, including Ppara, Cpt1, Acol and Mcad, were significantly upregulated in BAT of $\mathrm{Atf4}^{-/-}$compared with $\mathrm{Atf4^{+/+ }}$ mice (Figure $5 \mathrm{~A})$. Expression of the mitochondria marker, $\mathrm{Nrf} 2$, was also upregulated in these knockout (KO) mice (Figure $5 \mathrm{~A})$. In addition, levels of lipoprotein lipase $(\mathrm{Lpl})$, fatty acid transport protein (Fatp), fatty acid-binding protein (Fabp) and Cd36 mRNAs were also significantly increased in BAT of these mice (Figure 5B).

\section{Increased expression of UCP1 in BAT of Atf $4^{-/-}$mice}

Increased fatty acid oxidation is expected to be accompanied with higher levels of ATP production. By contrast, ATP levels in BAT were very low in $\mathrm{Atf4}^{-/}$ compared with $\mathrm{AtfH}^{+/+}$mice (data not shown). This is not caused by altered mitochondrial function, as enzymatic activities of complexes, including Complex I, Complex II and Complex III, were not different between Atf $4^{-1}$ and $\mathrm{Atfl}^{+/+}$mice (data not shown).

The main function of BAT is thermogenesis, which is mediated by upregulating the expression of UCP1. Levels of $U c p 1,2$ and 3 mRNA were significantly increased in BAT of $\mathrm{Atf4}^{-/}$compared with $\mathrm{Atf4}{ }^{+/+}$mice (Figure $5 \mathrm{C})$. Consistent with changes in mRNA levels, UCP1 protein levels were also increased in these mice (Figure 5D). Furthermore, oxygen consumption was also significantly increased in BAT isolated from $\mathrm{Atf}^{-/-}$mice (Figure $5 \mathrm{E})$. There was no significant difference, however, following stimulation with oleate (Figure 5E). In support of this, BAT seems to be much denser in $\mathrm{Atf4}^{-/-}$mice (Figure $5 F)$. Consistent with the higher expression of UCP1, the basal core body temperature of $\mathrm{Atf4}^{-/-}$mice was higher than that of wild-type mice (Figure 2F). Moreover, $A t f 4^{-/-}$mice displayed higher expression of UCP1 and core body temperature in response to cold stress (data not shown).

To investigate the mechanisms of increased UCP1 expression, we examined expression levels of transcription factors that regulate UCP1 gene expression, including PGC1 $\alpha$ and PPAR $\gamma[25,26]$. Expression of Pgcl $\alpha$ and Ppary was significantly increased in BAT of $\mathrm{Atf4}^{-/}$ mice compared with $\mathrm{Atf} 4^{+/+}$mice (Figure $5 \mathrm{G}$ ). Increase of UCP1 is regulated by the SNS through the activation of Adrb1 and Adrb3. Our results showed that levels of Adrb1 and Adrb3 mRNAs were significantly increased in BAT of $\mathrm{Atf4}^{-/-}$mice (Figure 5G).

\section{Discussion}

As previously reported, $\mathrm{Atf}^{4^{-/-}}$mice are smaller [16]. We noticed that, however, Atf $4^{-/}$mice were leaner and accumulated less fat in their WAT. The goal of our current study is to elucidate the molecular and cellular mechanisms underlying the reduced fat mass in $\mathrm{Atf4}^{-/-}$ mice. Our results suggest that the reduced fat mass in Atf $4^{-/-}$mice was caused by increased energy expenditure, rather than decreased food intake. Furthermore, the increased energy expenditure was due to increased ther- 
A

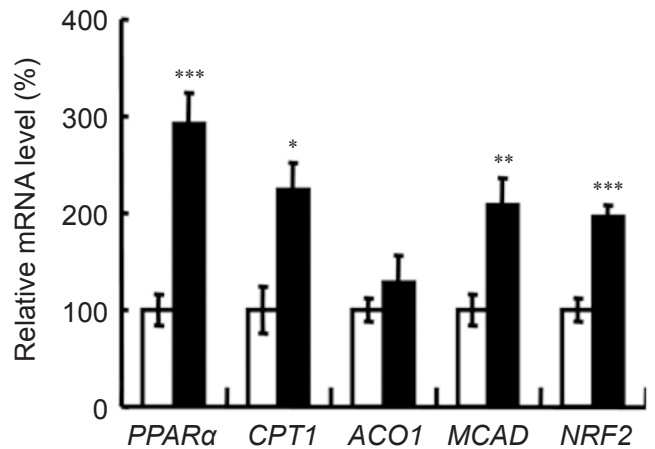

C

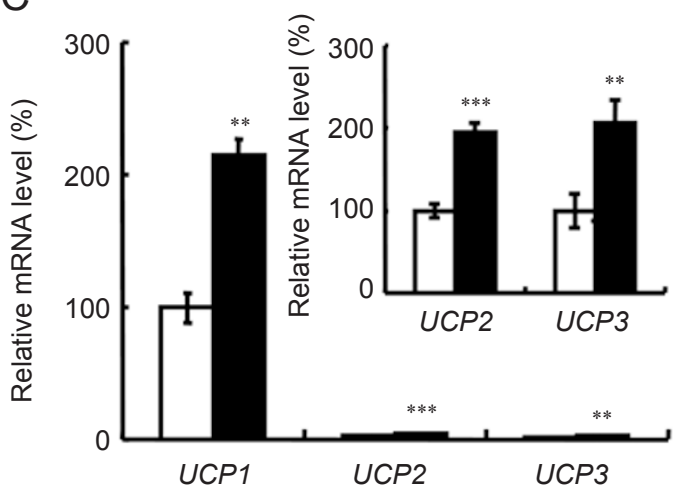

B
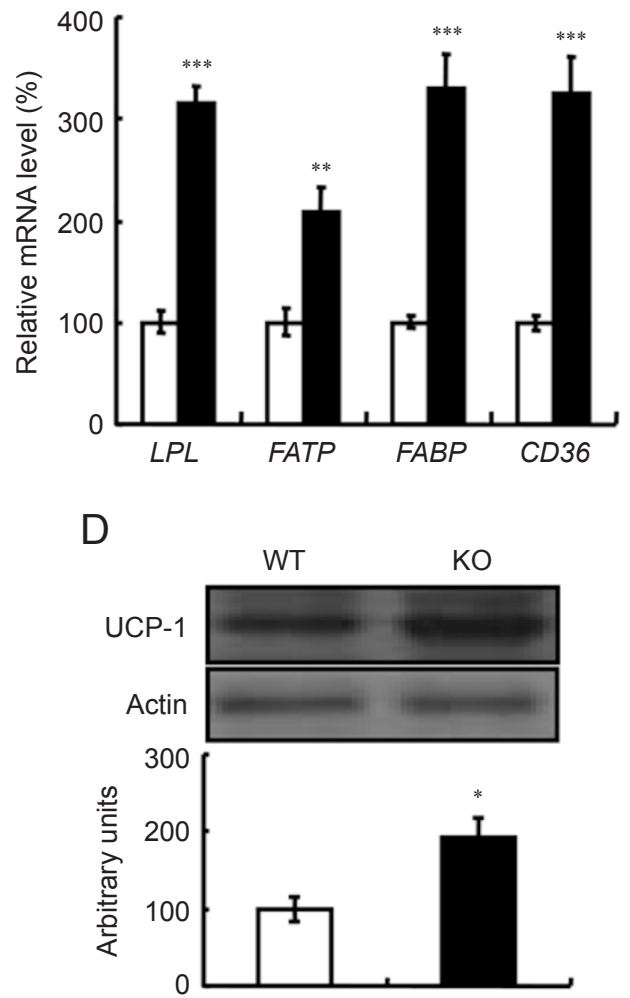

$E$

$\mathrm{F}$
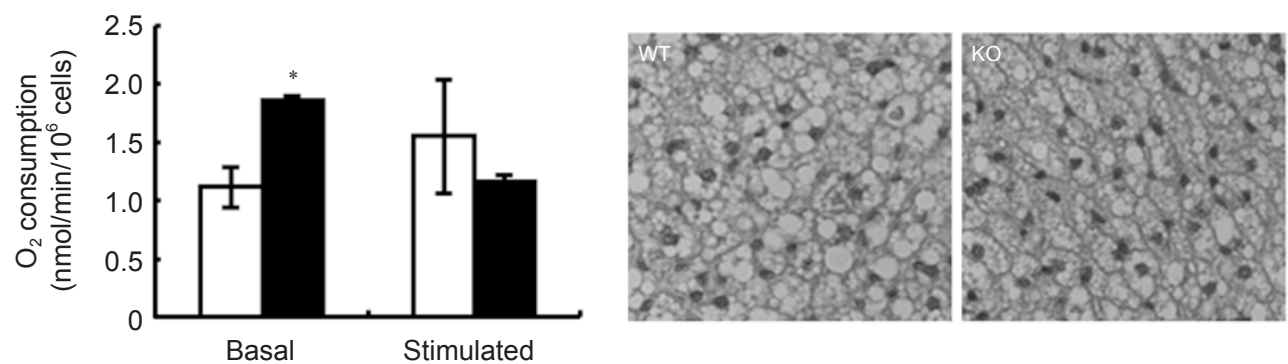

G

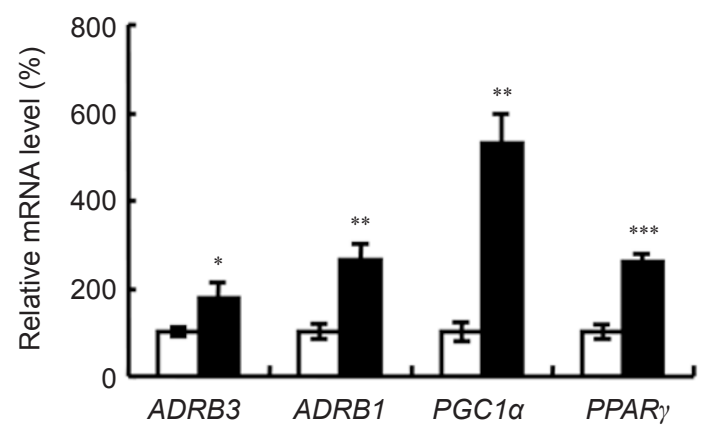

Figure 5 Expression of $\beta$-oxidation genes and UCP1 mRNA and protein increases in BAT of Atf $^{-1-}$ mice. Expression of $\beta$-oxidation genes, Ucp1 mRNA and protein in BAT. Data are mean \pm SEM of at least two independent western blot or real-time PCR experiments with mice, as indicated $\left(A t f 4^{+/+}\right.$mice $(n=8)$, Atf $4^{-1-}$ mice $\left.(n=8)\right)$. Statistical significance was determined by two-tailed student $t$-test $(* P<0.05, * * P<0.01, * * * P<0.001)$. (A) $P P A R a, C P T 1, A C 01, M C A D$ and NRF2 mRNA. (B) $L P L$, FATP, FABP and CD36 mRNA. (C) UCP1, UCP2 and UCP3 mRNA. (D) UCP1 protein (top, western blot; bottom, UCP1 relative to Actin). (E) Oxygen consumption in isolated BAT. (F) HE staining of BAT. (G) ADRB3, ADRB1, PGC1 $\alpha$ and PPAR mRNA. 
mogenesis, but not a difference in locomotor activity.

It is well known that ATF4 is involved in regulation of ER stress, osteoblast biology, amino acid metabolism and glucose metabolism [20, 21, 27]. There has been no report, however, showing that ATF4 regulates lipid metabolism. ATF4 belongs to the CREB family. Several studies have suggested a role for CREB in regulating lipid metabolism. For example, mice deficient in CREB develop fatty liver and display elevated expression of the nuclear hormone receptor PPAR $\gamma$ [28]. Furthermore, it has also been shown that CREB inhibits expression of SREBP1c, another key transcription factor regulating lipogenesis, and this effect is antagonized by ATF4 [29]. These studies suggest a possible involvement of ATF4 in regulating lipid metabolism. This hypothesis was confirmed by our current study, which provides in vivo evidence indicating a role for ATF4 in the control of lipid metabolism in WAT and thermogenesis in BAT.

Our results suggest that the decreased fat mass in Atf4 $^{-/-}$mice was caused by a reduction in cell volume rather than cell number. Since adipocyte volume is determined by the content of intracellular lipids [30], we hypothesized that reduction of adipocyte volume in $\mathrm{Atf4}^{-/-}$mice results from suppressed lipogenesis and increased mobilization of intracellular fat. Consistent with this possibility, protein levels of FAS and SREBP1c decreased significantly in WAT of $\mathrm{AtfH}^{-/}$mice, suggesting decreased lipogenesis. A previous in vitro study showed that ATF4 is a positive regulator of SREBP1c expression [29]. Despite the decreased protein levels, mRNA levels of Srebplc, however, are higher in WAT of $\mathrm{Atf4}^{-/-}$mice, suggesting that ATF4 may regulate Srebp1c expression at the translational level. FAS and SCD1 are direct target genes of SREBP1c. The low levels of FAS and SCD1 mRNAs are consistent with the low protein levels of SREBP1c in WAT of $\mathrm{Atf4}^{-/-}$mice.

Fat mobilization is normally mediated via increased activities of the SNS [31, 32]. Increased release of NE from sympathetic nerves activates Adrb3 on the surface of adipocytes, increasing intracellular levels of cAMP that activates PKA, which mediates phosphorylation and activation of HSL [33, 34]. Increased expression of Atgl and Perilipin is also required for fat mobilization $[33,34]$. Levels of p-HSL, as well as Atgl and Perilipin $A$ expression were upregulated in WAT of $A t f 4^{-/-}$ mice, suggesting an increase in lipolysis in these mice. The glycerol release following stimulation with the $\beta$-adrenoceptor agonist isoproterenol was significantly higher (5-fold) in WAT isolated from $\mathrm{Atf4}^{-/-}$compared with Atf $^{+/+}$mice, suggesting an increased capacity for lipolysis in response to NE in these mice. In fact, levels of serum NE were much higher in $\mathrm{Atf4}^{-/-}$mice, suggest- ing that the increased lipolysis is mediated by increased activities of SNS in these mice. In addition to the SNS, fat mobilization can also be regulated by hormones, such as insulin, which acts to inhibit lipolysis. Levels of serum insulin, however, are unaffected in $\mathrm{Atf4}^{-/-}$compared with $A t f 4^{+/+}$mice. Further investigation will be required to determine the relative contributions of the SNS and other hormones in the regulation of increased fat mobilization in $\mathrm{Atf4}^{-/-}$mice. Taken together, these results suggest that reduced fat mass in $A t f 4^{-/-}$mice is caused by suppression of fatty acid synthesis and activation of TG lipolysis. These changes are consistent with the observed low RER, which implies that fat is the major source of energy in $\mathrm{Atf4}^{-/}$mice.

Because of the lower levels of serum TG and FFA, we speculated that FFA are either oxidized in WAT or secreted into circulation and metabolized by other tissues, such as liver, skeletal muscle or BAT. Expression of $\beta$-oxidation-related genes was upregulated in WAT and BAT, but not in liver and muscle, in $\mathrm{Atf4}^{-/-}$compared with $\mathrm{AtfH}^{+/+}$mice. As fatty acid oxidation in WAT makes only a minor contribution to removing fatty acids [35], we hypothesized that increased $\beta$-oxidation in BAT makes primary contributions to utilize fatty acids and decrease serum fatty acid levels in $\mathrm{Atf4}^{-/-}$mice. Consistent with this possibility, genes encoding proteins involved in fatty acid uptake, including $\mathrm{Lpl}$, Fabp, Fatp and Cd36, were increased significantly in BAT of $\mathrm{Atf4}^{-/-}$compared with $\mathrm{Atf4}{ }^{+/+}$mice.

BAT is of major importance in the regulation of thermogenesis and energy expenditure, which is achieved by increasing expression of UCP1 $[7,36]$. The role of UCP1 in thermogenesis-regulated body weight control is demonstrated by the development of obesity in UCP1-KO mice [8]. Moreover, upregulation of UCP1 expression results in increased thermogenesis and energy expenditure, which helps to protect from fat accumulation and obesity [37]. In the current study, we showed that levels of UCP1 mRNA and protein increased significantly in BAT of $\mathrm{Atf4}^{-/-}$mice. Consistent with these changes, oxygen consumption in isolated BAT was also increased in Atf $4^{-/}$mice. Since there was no difference in locomotor activities between $A t f 4^{-/-}$and $A t f 4^{+/+}$mice, the increased thermogenesis accounts for the major part of increased energy expenditure in $\mathrm{Atf4}^{-/-}$mice. Consistent with this, rectal temperature of $\mathrm{Atf4^{-/- }}$ mice was consistently higher throughout the 24-h period at the basal level, as well as in response to cold stress.

Expression of UCP1 is under complex control by the SNS and hormones including NE, EPI and T3. Atf $4^{-1-}$ mice exhibit no difference in serum EPI and T3 levels compared with $\mathrm{AtfH}^{+/+}$mice. Levels of serum NE, how- 
ever, were significantly increased in $\mathrm{Atf}^{-/-}$mice, suggesting a possible involvement of the SNS in enhanced $\mathrm{UCP} 1$ expression. It has been reported that Ucp 1 expression, in response to activation of the SNS, is upregulated by the transcription factors PGC1 $\alpha$ and PPAR $\gamma[25,26]$. $P g c 1 \alpha$ and Ppary expression were also increased in BAT of $\mathrm{Atf}^{-/-}$mice, suggesting that the increased UCP1 expression is mediated by PGC1 $\alpha$ and PPAR $\gamma$. Alternatively, expression of UCP1 could also be activated by increased intracellular fatty acid levels [7]. Consistent with this possibility, genes encoding fatty acid transport proteins were upregulated in BAT of $\mathrm{Atf}^{-/-}$mice.

While our manuscript was being prepared, Graff and co-workers [24] reported a potential role of ATF4 in regulating metabolism in invertebrates and mammals. Consistent with their findings, we also found that $\mathrm{Atf}^{-/-}$mice are lean, have increased energy expenditure and display higher core temperature. There are different results, however, regarding serum glucose and insulin levels, as well as UCP1 expression in WAT and BAT of $\mathrm{Atf4}^{-/-}$mice, between our and their work. They, and another group [38], reported that $A t f 4^{-/}$mice are hypoglycemic with higher level of insulin [24]. By contrast, we did not observe any difference in serum insulin and glucose levels in 8-12-week-old $\mathrm{Atf4}^{-/-}$mice compared with their wildtype control mice. Furthermore, they showed that UCP1 expression is much higher in WAT, but not in BAT, of Atf $4^{-/-}$mice [24]. By contrast, we found that UCP1 expression increases significantly in BAT, but not in WAT, in $\mathrm{Atf}^{-/-}$mice. We consider the difference could be due to the different background of mice used in our and their studies. In addition, we also analyzed changes of lipid metabolism in WAT of $\mathrm{Atf}^{-/-}$mice and found that ATF4 plays a key role in regulating lipolysis, fatty acid oxidation and lipogenesis.

In summary, we show that the decreased fat mass in $\mathrm{Atf}^{-/-}$mice is caused by increased fat mobilization and suppressed fatty acid synthesis in WAT, as well as increased thermogenesis in BAT by increasing the expression of UCP1. Taken together, our study identifies a possible new function for ATF4 in regulating lipid metabolism and thermogenesis. Increased energy expenditure caused by ATF4 deletion provides a rationale for developing drugs targeting ATF4 for treatment of obesity and associated metabolic diseases.

\section{Materials and Methods}

\section{Animals}

Heterozygous $\left(\mathrm{Atf}^{+/-}\right)$mice on $129 \mathrm{SV}$ background were kindly provided by Dr Tim Townes (University of Alabama at Birmingham) [16]. Heterozygous $\left(A t f 4^{+/}\right)$mice were bred to produce homozygous $\left(A t f 4^{-/}\right)$and wild-type $\left(A t f 4^{+/}\right)$mice and genotyping were performed as previously described [39]. Male $\mathrm{Atft}^{+/+}$mice and $A t f 4^{-/}$mice (2-3-month-old) were maintained on a $12 \mathrm{~h} \mathrm{light/}$ dark cycle at $25^{\circ} \mathrm{C}$, and were provided free access to commercial rodent chow and tap water prior to the experiments. Food intake and body weight were recorded daily. Animals were killed by $\mathrm{CO}_{2}$ inhalation, and trunk blood was collected for the assays described below. Body and tissue weights were recorded at the time of killing. WAT and BAT were isolated and put into $4 \%$ paraformaldehyde buffer immediately for histological study, as described later. All animal experimental procedures were reviewed and approved by the Institutional Animal Care and Use Committee of Institution for Nutritional Sciences.

\section{Indirect calorimetry}

Male mice (2-3-month-old) were maintained in a comprehensive lab animal monitoring system (Columbus Instruments, Columbus, OH, USA) for $24 \mathrm{~h}$ according to the instructions of the manufacturer. After the mice adapted to the metabolic chamber for $6 \mathrm{~h}$, volume of $\mathrm{O}_{2}$ consumption and $\mathrm{CO}_{2}$ production were continuously recorded over 24-h period.

\section{Rectal temperature measurement}

The rectal temperatures of the mice were measured using a rectal probe attached to a digital thermometer (Physitemp Inc, NJ, USA).

\section{Serum measurements}

Serum was obtained by centrifugation of clotted blood and then snap-frozen in liquid nitrogen and stored at $-20{ }^{\circ} \mathrm{C}$. Serum TG, total cholesterol and free fatty acid levels were determined enzymatically using TG reagent (wako, Japan), Infinity cholesterol reagent (wako) and NEFA C reagent (wako), respectively. Serum NE, T3 and EPI levels were determined using ELISA Kits from R\&D. All of these assays were performed according to manufacturers instructions.

\section{Histological analysis of WAT and BAT}

BAT and WAT were fixed in $4 \%$ paraformaldehyde overnight and stained with hematoxylin and eosin, as previously described [40]. The WAT cell volumes were analyzed as described previously [41].

\section{BAT in vitro oxygen consumption measurement}

Brown adipocytes were isolated and oxygen consumption was measured using a Clark-type oxygen electrode (Hansatech Instruments Ltd, Norfolk, England), as previously described [7], with minor modifications. Each sample was analyzed by incubating $1 \times$ $10^{6}$ cells in a magnetically stirred chamber at $37^{\circ} \mathrm{C}$. After the basal respiration was recorded, $5 \mathrm{mM}$ oleate was added to determine the maximal oxygen consumption.

\section{Glycerol release assay}

WAT was removed and incubated in Krebs-Ringer Hepes buffer (KRHB) containing $1 \mathrm{mg} / \mathrm{ml}$ collagenase (Sigma) and $2 \%$ BSA, as previously described [42]. Freshly isolated adipocytes were incubated in KRHB containing adenosine deaminase (Sigma) in the absence or presence of $\beta$-adrenergic agonist isoproterenol $(1 \mu \mathrm{M})$. Lipolysis was assessed as the release of glycerol in the 
cultured medium, as described previously [43], with free glycerol reagent (Sigma-Aldrich, St-Louis, MO, USA).

\section{$R N A$ isolation and relative quantitative $R T-P C R$}

Total RNA was prepared from mouse tissues by using TRIzol reagent (Invitrogen). RNA $(2 \mu \mathrm{g})$ was reverse transcribed with random primers (Invitrogen) and M-MLV Reverse Transcriptase (Invitrogen). Quantitative PCR was performed with a qPCR core kit for SYBR green I (ABI) in an ABI 7500 system (Applied Biosystem). PCR products were subjected to a melting curve analysis. At a specific threshold in the linear amplification stage, the cycle differences between amplified GAPDH (as an internal control) and cDNAs of interest were used to determine the relative expression levels of the genes of interest. Primer sequences used in this study are available on request.

\section{Western blot analysis}

Whole-cell lysates from frozen tissues were isolated using RIPA lysis buffer (150 mM Tris-HCl, $50 \mathrm{mM} \mathrm{NaCl,} \mathrm{1 \%} \mathrm{NP-40,}$ $0.1 \%$ tween-20). Protease and phosphatase inhibitors were added to all buffers before experiments. Protein concentration was assayed using BCA protein assay (Pierce, Rockford, IL, USA). Primary antibodies (anti-FAS antibody (BD Scientific), anti-CPT1, anti-p-HSL, anti-PPAR $\alpha$, anti-PKA substrates and anti-HSL antibodies (Cell Signaling), anti-SREBP1c and anti-UCP1 antibody (Santa Cruz Biotechnology)) were incubated overnight at $4{ }^{\circ} \mathrm{C}$ and specific proteins were visualized by ECL Plus (Amersham Biosciences). Band intensities were measured using ImageQuant (BioRad Laboratories) and normalized to $\beta$-actin.

\section{Data analysis}

All data are expressed as mean \pm SEM for experiments and numbers of mice used as indicated. The two-tailed Student's $t$-test was used to evaluate statistical differences between $\mathrm{Atf}^{+/+}$and Atf $4^{-/}$mice (two-tailed Student's $t$-test, $* P<0.05$, $* * P<0.01$, $* * * P<0.001)$. Means for all parameters examined in current study were calculated independently for male mice. No statistical difference in the parameters examined between male and female mice was observed (two-tailed Student's $t$-test, $P>0.05$ ).

\section{Acknowledgment}

This work was supported by grants from the Ministry of Science and Technology of China (973 Program 2009CB919001 and 2010CB912502), the National Natural Science Foundation (30871208 and 30890043), the Chief Scientist Program of Shanghai Institutes for Biological Sciences, the Chinese Academy of Sciences (SIBS2008006) and Novo Nordisk-CAS Research Foundation (NNCAS-2008-10 [INS,SIBS]). Dr Feifan Guo was also supported by the One Hundred Talents Program of the Chinese Academy of Scences and the Pujiang Talents Program of Shanghai Municipality (08PJ14107).

\section{References}

1 Rosen ED, Spiegelman BM. Adipocytes as regulators of energy balance and glucose homeostasis. Nature 2006; 444:847-
853.

2 Spiegelman BM, Flier JS. Obesity and the regulation of energy balance. Cell 2001; 104:531-543.

3 Penicaud L, Cousin B, Leloup C, Lorsignol A, Casteilla L. The autonomic nervous system, adipose tissue plasticity, and energy balance. Nutrition 2000; 16:903-908.

4 Kopecky J, Rossmeisl M, Hodny Z, Syrovy I, Horakova M, Kolarova P. Reduction of dietary obesity in aP2-Ucp transgenic mice: mechanism and adipose tissue morphology. Am J Physiol 1996; 270:E776-E786.

5 Ricquier D, Bouillaud F. The uncoupling protein homologues: UCP1, UCP2, UCP3, StUCP and AtUCP. Biochem J 2000; 345 Pt 2:161-179.

6 Enerback S, Jacobsson A, Simpson EM, et al. Mice lacking mitochondrial uncoupling protein are cold-sensitive but not obese. Nature 1997; 387:90-94.

7 Matthias A, Ohlson KB, Fredriksson JM, Jacobsson A, Nedergaard J, Cannon B. Thermogenic responses in brown fat cells are fully UCP1-dependent. UCP2 or UCP3 do not substitute for UCP1 in adrenergically or fatty scid-induced thermogenesis. J Biol Chem 2000; 275:25073-25081.

8 Feldmann HM, Golozoubova V, Cannon B, Nedergaard J. UCP1 ablation induces obesity and abolishes diet-induced thermogenesis in mice exempt from thermal stress by living at thermoneutrality. Cell Metab 2009; 9:203-209.

9 Xue B, Coulter A, Rim JS, Koza RA, Kozak LP. Transcriptional synergy and the regulation of Ucp1 during brown adipocyte induction in white fat depots. Mol Cell Biol 2005; 25:8311-8322.

10 Kalaany NY, Gauthier KC, Zavacki AM, et al. LXRs regulate the balance between fat storage and oxidation. Cell Metab 2005; 1:231-244.

11 Mielnicki LM, Pruitt SC. Isolation and nucleotide sequence of a murine cDNA homologous to human activating transcription factor 4. Nucleic Acids Res 1991; 19:6332.

12 Chevray PM, Nathans D. Protein interaction cloning in yeast: identification of mammalian proteins that react with the leucine zipper of Jun. Proc Natl Acad Sci USA 1992; 89:57895793.

13 Karpinski BA, Morle GD, Huggenvik J, Uhler MD, Leiden JM. Molecular cloning of human CREB-2: an ATF/CREB transcription factor that can negatively regulate transcription from the cAMP response element. Proc Natl Acad Sci USA 1992; 89:4820-4824.

14 Vallejo M, Ron D, Miller CP, Habener JF. C/ATF, a member of the activating transcription factor family of DNA-binding proteins, dimerizes with CAAT/enhancer-binding proteins and directs their binding to cAMP response elements. Proc Natl Acad Sci USA 1993; 90:4679-4683.

15 Mamady H, Storey KB. Coping with the stress: expression of ATF4, ATF6, and downstream targets in organs of hibernating ground squirrels. Arch Biochem Biophys 2008; 477:77-85.

16 Masuoka HC, Townes TM. Targeted disruption of the activating transcription factor 4 gene results in severe fetal anemia in mice. Blood 2002; 99:736-745.

17 Bartsch D, Ghirardi M, Casadio A, et al. Enhancement of memory-related long-term facilitation by ApAF, a novel transcription factor that acts downstream from both CREB1 and CREB2. Cell 2000; 103:595-608. 
18 Brightwell JJ, Gallagher M, Colombo PJ. Hippocampal CREB1 but not CREB2 is decreased in aged rats with spatial memory impairments. Neurobiol Learn Mem 2004; 81:19-26.

19 Mohamed HA, Yao W, Fioravante D, Smolen PD, Byrne JH. cAMP-response elements in Aplysia creb1, creb2, and Ap-uch promoters: implications for feedback loops modulating long term memory. J Biol Chem 2005; 280:27035-27043.

20 Yang X, Matsuda K, Bialek P, et al. ATF4 is a substrate of RSK2 and an essential regulator of osteoblast biology; implication for Coffin-Lowry Syndrome. Cell 2004; 117:387-398.

21 Ohoka N, Yoshii S, Hattori T, Onozaki K, Hayashi H. TRB3, a novel ER stress-inducible gene, is induced via ATF4CHOP pathway and is involved in cell death. EMBO J 2005; 24:1243-1255.

22 Whitney ML, Jefferson LS, Kimball SR. ATF4 is necessary and sufficient for ER stress-induced upregulation of REDD1 expression. Biochem Biophys Res Commun 2009; 379:451455.

23 Siu F, Bain PJ, LeBlanc-Chaffin R, Chen H, Kilberg MS. ATF4 is a mediator of the nutrient-sensing response pathway that activates the human asparagine synthetase gene. $J$ Biol Chem 2002; 277:24120-24127.

24 Seo J, Fortuno ES 3rd, Suh JM, et al. Atf4 regulates obesity, glucose homeostasis, and energy expenditure. Diabetes 2009; 58:2565-2573.

25 Handschin C, Spiegelman BM. Peroxisome proliferatoractivated receptor gamma coactivator 1 coactivators, energy homeostasis, and metabolism. Endocr Rev 2006; 27:728-735.

26 Yubero P, Manchado C, Cassard-Doulcier AM, et al. CCAAT/ enhancer binding proteins alpha and beta are transcriptional activators of the brown fat uncoupling protein gene promoter. Biochem Biophys Res Commun 1994; 198:653-659.

27 Yamaguchi S, Ishihara H, Yamada T, et al. ATF4-mediated induction of 4E-BP1 contributes to pancreatic beta cell survival under endoplasmic reticulum stress. Cell Metab 2008; 7:269276.

28 Herzig S, Hedrick S, Morantte I, Koo SH, Galimi F, Montminy M. CREB controls hepatic lipid metabolism through nuclear hormone receptor PPAR-gamma. Nature 2003; 426:190193.

29 Zhou L, Li Y, Nie T, et al. Clenbuterol inhibits SREBP-1c expression by activating CREB1. J Biochem Mol Biol 2007; 40:525-531.

30 Goldrick RB. Morphological changes in the adipocyte during fat deposition and mobilization. Am J Physiol 1967; 212:777-
782.

31 Lowell BB, Spiegelman BM. Towards a molecular understanding of adaptive thermogenesis. Nature 2000; 404:652660.

32 Nogueiras R, Perez-Tilve D, Veyrat-Durebex C, et al. Direct control of peripheral lipid deposition by CNS GLP-1 receptor signaling is mediated by the sympathetic nervous system and blunted in diet-induced obesity. J Neurosci 2009; 29:59165925.

33 Holm C, Osterlund T, Laurell H, Contreras JA. Molecular mechanisms regulating hormone-sensitive lipase and lipolysis. Annu Rev Nutr 2000; 20:365-393.

34 Egan JJ, Greenberg AS, Chang MK, Wek SA, Moos MC Jr, Londos C. Mechanism of hormone-stimulated lipolysis in adipocytes: translocation of hormone-sensitive lipase to the lipid storage droplet. Proc Natl Acad Sci USA 1992; 89:8537-8541.

35 Frayn KN, Langin D, Karpe F. Fatty acid-induced mitochondrial uncoupling in adipocytes is not a promising target for treatment of insulin resistance unless adipocyte oxidative capacity is increased. Diabetologia 2008; 51:394-397.

36 Wolf G. Brown adipose tissue: the molecular mechanism of its formation. Nutr Rev 2009; 67:167-171.

37 Li B, Nolte LA, Ju JS, et al. Skeletal muscle respiratory uncoupling prevents diet-induced obesity and insulin resistance in mice. Nat Med 2000; 6:1115-1120.

38 Yoshizawa T, Hinoi E, Jung DY, et al. The transcription factor ATF4 regulates glucose metabolism in mice through its expression in osteoblasts. J Clin Invest 2009; 119:2807-2817.

39 Tanaka T, Tsujimura T, Takeda K, et al. Targeted disruption of ATF4 discloses its essential role in the formation of eye lens fibres. Genes Cells 1998; 3:801-810.

40 Carson FL. Histotechnology: A Self-Instructional Text. 2nd edn. Chicago: American Society for Clinical Pathology Press, 1997.

41 Gerin I, Dolinsky VW, Shackman JG, et al. LXRbeta is required for adipocyte growth, glucose homeostasis, and beta cell function. J Biol Chem 2005; 280:23024-23031.

42 Wang SP, Laurin N, Himms-Hagen J, et al. The adipose tissue phenotype of hormone-sensitive lipase deficiency in mice. Obes Res 2001; 9:119-128.

43 Miyoshi H, Souza SC, Zhang HH, et al. Perilipin promotes hormone-sensitive lipase-mediated adipocyte lipolysis via phosphorylation-dependent and -independent mechanisms. $J$ Biol Chem 2006; 281:15837-15844.

(Supplementary information is linked to the online version of the paper on the Cell Research website.) 